See discussions, stats, and author profiles for this publication at: https://www.researchgate.net/publication/339579515

\title{
Wave Attenuation Service by Intertidal Coastal Ecogeosystems in the Bay of Mont-Saint-Michel, France: Review and Meta-Analysis
}

Chapter · January 2020

DOI: 10.1007/978-981-15-2081-5_32

\section{CITATIONS}

0

4 authors:

Antoine Mury

Ecole Pratique des Hautes Etudes

12 PUBLICATIONS 10 CITATIONS

SEE PROFILE

Samuel Etienne

Ecole Pratique des Hautes Etudes

160 PUBLICATIONS 1,388 CITATIONS

SEE PROFILE

Some of the authors of this publication are also working on these related projects:

Fanzines et autres publications DIY View project

Project Geomorphoclim: Paraglacial dynamics in Svalbard View project
READS

59

Antoine Collin

Ecole Pratique des Hautes Etudes

82 PUBLICATIONS 839 CITATIONS

SEE PROFILE

Matthieu Jeanson

Centre Universitaire de Mayotte

29 PUBLICATIONS 124 Citations

SEE PROFILE 


\title{
Wave Attenuation Service By Intertidal Coastal Ecogeosystems In the Bay Of Mont-Saint-Michel, France: Review And Meta-analysis
}

\author{
Antoine Mury ${ }^{1 *}$, Antoine Collin' ${ }^{1}$, Samuel Etienne ${ }^{1}$ \\ ${ }^{I}$ Ecole Pratique des Hautes Etudes (EPHE), PSL University, CNRS LETG. \\ Matthieu Jeanson ${ }^{2}$ \\ ${ }^{2}$ Université de Mayotte, CNRS ESPACE-DEV.
}

\begin{abstract}
The wave attenuation service (WAS) literature review over the Bay of Mont-Saint-Michel encompasses saltmarshes, mudflats, seagrasses, shelly cheniers, oyster, honeycomb worm and sandworm reefs, which all together will be named "ecogeosystems" in this review due to their combination of biogenic systems, ecological systems and geomorphological systems (Mury et al., 2018). It provides a comparative meta-analysis of the various measurements of wave attenuation induced by "ecogeosystems" across different studies focusing on wave height monitoring. The array of "ecogeosystems" included in this review was firstly identified through a fine-scale mapping of the Bay of Mont-SaintMichel, then literature research was led using several keywords related to ecosystem services and coastal protection. A total of 32 studies was compiled over six countries. The majority of studies was located on North-Atlantic Ocean coastlines. According to their specificities, "ecogeosystems" were synthetically split into two categories, surface (saltmarshes, mudflats, seagrasses and oyster reefs) and punctual (shelly cheniers), for the sake of the WAS comparability. WAS associated with surface saltmarshes, mudflats, seagrasses, and oyster reefs ranged from 0.25 to $7.86 \% / \mathrm{m}$, from 0.01 to $4 \% / \mathrm{m}$, from 0.03 to $0.77 \% / \mathrm{m}$, from 1 to $64 \% / \mathrm{m}$, respectively. The punctual shelly chenier was monitored from 70 to $98 \%$. Honeycomb worm and sandworm reefs have no data about WAS according to our literature review.
\end{abstract}

Key words: Protection Ecosystem Service; Coastal Hazards; Coastal Management; Mega-tidal Environment.

*Corresponding author. Email: antoine.mury@etu.ephe.psl.eu

\section{Introduction}

A consequence of global change might be an increase in coastal risks (Temmerman et al., 2013). Indeed, sea-level rise conjugated with spring tides, can lead to hazardous sealevels, in worldwide coastal areas subject to unprecedented demographic densification (exposure), thus risks. Coastal "ecogeosystems" such as tropical coral reefs, have the potential to alleviate hazards by reducing wave energy due to their topographic complexity (drag coefficient, Harris et al., 2016, 2018). However, studies focusing on the wave attenuation service (WAS) of natural barriers remain spatially discrete and scattered, as well as not harmonized. Moreover, the WAS of some barrier types remains poorly known, or even unknown.

A comprehensive view of the ensemble of coastal "ecogeosystems" is needed to help stakeholders tasked with coastal risk reduction and coastal protection. The Bay of Mont-Saint-Michel (BMSM, France) suitably matches this objective, given its mega-tidal regime and the large panel of natural barriers (Collin et al., 2018a) facing hazards and protecting human assets.
The WAS literature review over the BMSM encompasses saltmarshes, mudflats, seagrasses, shelly cheniers, oyster, honeycomb worm and sandworm reefs. It offers a comparative view of the various values of wave attenuation by "ecogeosystems" observed across different studies through wave height monitoring. Ecological parameters acting as wave height attenuation factors have also been evaluated. Beyond the comparative results, the main objective of the study is to give an overview of the existing knowledge of WAS evaluation and identify the gaps hindering an efficient implementation of ecogeosystem-based solution to sustainably cope with coastal risks. Therefore, this work will be an opportunity to bring new data on the WAS of shelly cheniers, honeycomb worm and sandworm reefs, three types of "ecogeosystems" still under-considered for their role in coastal protection (only two studies: Jeanson et al., 2016; Mury et al., 2018).

\section{Methods}

The first step of this review work consists in the identification of the various "ecogeosystems" of the intertidal environment, which may take part in the wave mitigation process and thus which have to be considered in 
an integrated vision towards the management of coastal risks.

In so doing, five different "ecogeosystems" of the intertidal domain, which can possibly take part of the WAS, were able to be identified from a mapping of the BMSM (saltmarshes, mudflats/sandflats, shelly cheniers, honeycomb worm and sandworm reefs) (Figs 1, 2, 3), and two others were identified in the literature (seagrasses, and oyster reefs) to have a more complete view of the intertidal "ecogeosystems" of temperate coastal areas.

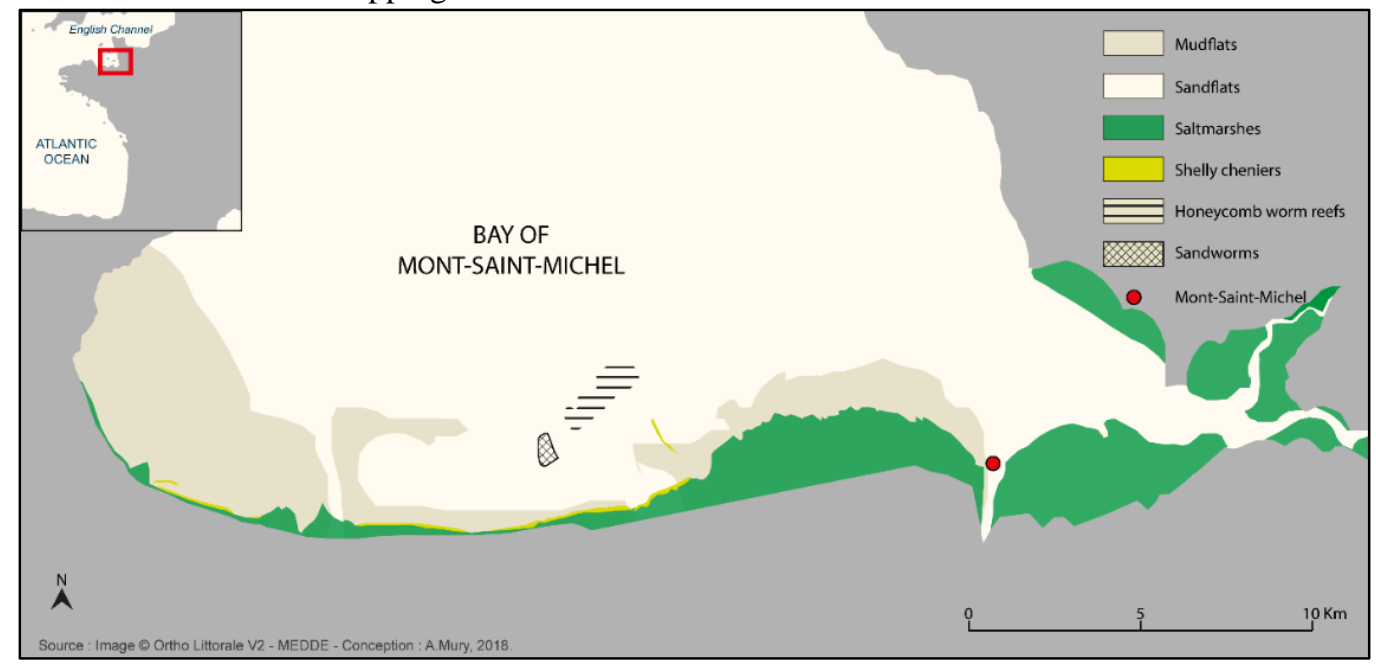

Figure 1. Location map of the "ecogeosystems" observed in the Bay of Mont-Saint-Michel, France

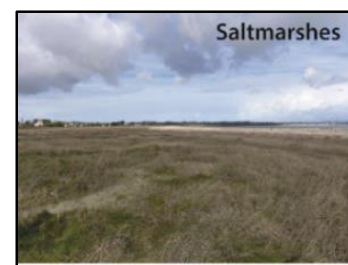

Honeycomb worm reefs
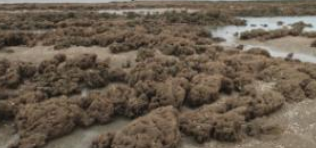

क्य

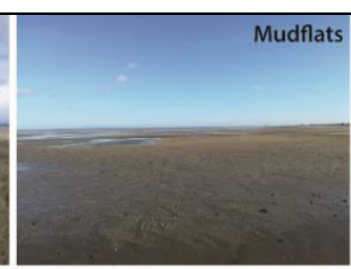

Sandworms beds

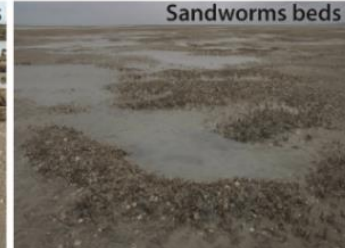

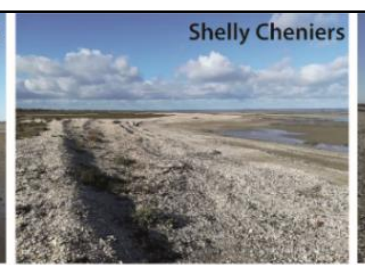
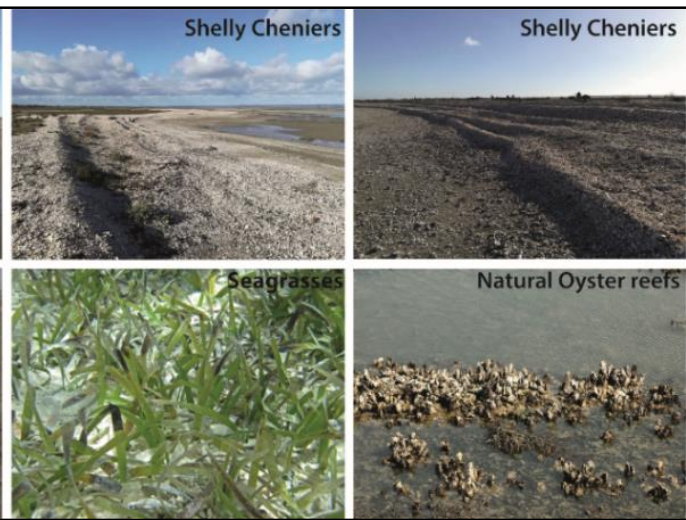

Figure 2. Illustration of the "ecogeosystems" concerned by the WAS review

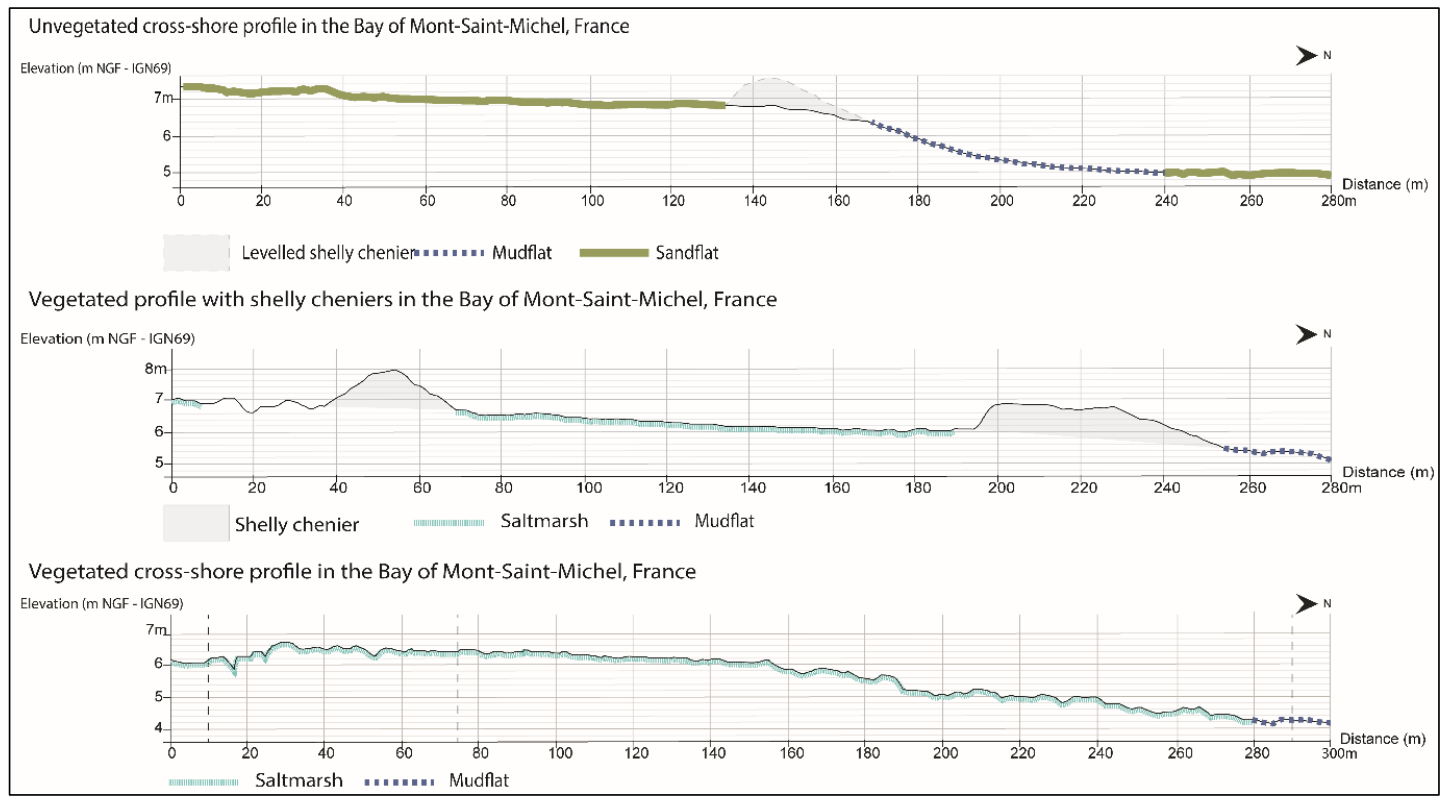

Figure 3. Cross-shore profiles in the Bay of Mont-Saint-Michel, France 
These "ecogeosystems" can be separated in three categories depending on their nature: (1) plant ecosystems (saltmarshes, seagrasses), (2) geomorphological structures (mudflats/sandflats, shelly cheniers), and (3) biogenic structures (honeycomb worm, oyster and sandworm reefs).

Concerning the screening method for scientific articles on WAS by coastal "ecogeosystems", the attention was focused specifically on the wave attenuation through the wave height attenuation data (WHA) (neither the velocity nor flow), due to its recognized representativeness of the submersion hazards. We examined the temperate intertidal natural features (what excluded the mangroves and coral reefs, which are tropical ecosystems as well as artificial seagrasses). We searched for the literature using research portals like Google Scholar, Web of Sciences and BibCnrs

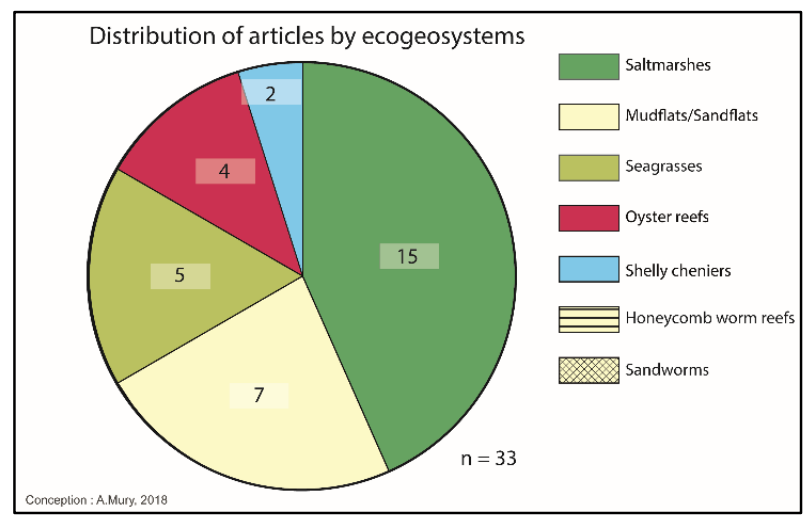

Figure 4. Diagram of the distribution of the papers
(Database of CNRS research teams) to target articles dealing with coastal protection, wave attenuation, ecosystem services and nature-based solutions for wave mitigation.

To achieve this literature review, several keywords were used, from generic keywords (i.e., ecosystem services, coastal protection service, coastal protection) to more detailed terms, in the form of feature + wave attenuation (or mitigation or reduction) or feature + wave height attenuation or feature + protection service (e.g., saltmarsh wave attenuation, saltmarsh wave height attenuation, saltmarsh protection service). Subsequently, were selected articles mentioning a percentage of attenuation by the whole selected features or a percentage per unit of distance $(\% / \mathrm{m})$ for the sake of data comparisons.

\section{Results}

A suite of 33 articles corresponded to the mentioned research criteria: 15 concerned saltmarshes WAS, 7 mudflats/sandflats, 5 seagrasses, 4 oyster reefs, 2 shelly cheniers, 0 dealt with honeycomb and sandworm reefs WAS. Some of the identified "ecogeosystems" like honeycomb worm reefs were not considered for their WAS yet, thus were not mentioned in the literature (Figure 4). Some "ecogeosystems", such as oyster reefs, are well known to act as natural breakwaters, but their WAS is seldom assessed. Most studies, extracted in this literature review, took place on the margins of the Atlantic Ocean, especially along the European and North-American coastlines (Figure 5).

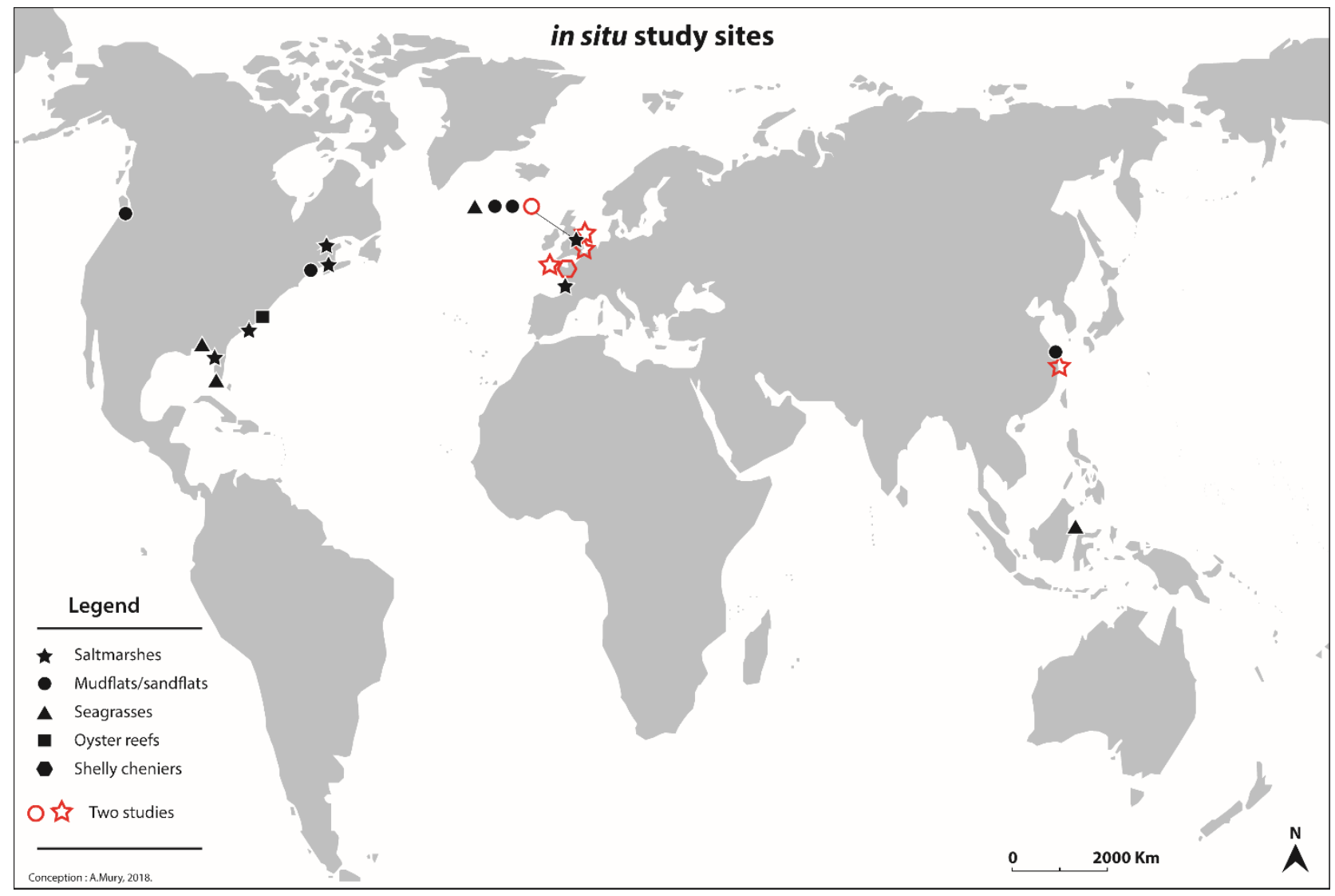

Figure 5. Location map of the in situ study sites, from the review 


\section{Saltmarshes}

About the saltmarsh review, fourteen studies dealing with the WAS were selected (Table 1). Considering the diversity across the different sites and the discrepancy in measurement methods in the literature dataset, we had to implement methodological choices to analyse the distinct study results. First, we decided to compare the studies by using the wave height attenuation (WHA) value, expressed as a percentage of attenuation per meter, to obtain a common unit between studies. When the WHA value was not directly provided by the authors, we calculated it (see shaded boxes). Even though the influence of the distance from the shore, of the foreshore and seabed characterization, the hydrodynamic conditions and water depth, underlying the studies was not accounted for, the calculated values offer a first-level comparison set. Second, we carried out a classification based on ecological criteria and more specifically on the plant length, to compare the
WAS across the studies. In this way, we retrieved plant communities with a higher length range (Spartina alterniflora, Spartina maritima, Scirpus mariqueter, Spartina patens), which corresponded to the fringing marsh of the lower mixed marsh communities (North-West European mixed saltmarsh, Elymus athericus, Puccinellia maritima, Salicornia, Suaeda maritima, etc.).

Regarding the higher plant community studies (Wayne 1976; Knutson et al., 1982; Morgan et al., 2009; Yang et al., 2008; Yang et al., 2012; Lechevalier, 2016), the WHA ranged from 0.95 to $7.86 \% / \mathrm{m}$ (mean: $3.3 \% / \mathrm{m}$ ).

These findings differed considerably from those observed for the lower mixed saltmarshes (Moller et al., 1996, 1999, 2002, 2006, 2014; Cooper, 2005; Collin et al., 2018). Indeed, the WHA measured bottomed at 0.3 and topped at $1.8 \% / \mathrm{m}$ (mean: $0.52 \% / \mathrm{m}$ ) range.

Table 1. Synthesis table of Saltmarshes wave attenuation service studies (WHA: Wave Height Attenuation; WEA: Wave Energy Attenuation; * grey literature, PS.: pressure sensors)

\begin{tabular}{|c|c|c|c|c|c|c|c|c|c|}
\hline \multirow{3}{*}{ Reference } & \multirow{3}{*}{$\begin{array}{c}\text { Study } \\
\text { Location }\end{array}$} & \multirow{3}{*}{$\begin{array}{l}\text { Tidal } \\
\text { Catego } \\
\text { ry }\end{array}$} & \multirow{3}{*}{$\begin{array}{c}\text { Material } \\
\mathbf{s}\end{array}$} & \multirow{3}{*}{\multicolumn{2}{|c|}{$\begin{array}{c}\text { Site } \\
\text { Characteristics }\end{array}$}} & \multicolumn{4}{|c|}{ Wave Attenuation } \\
\hline & & & & & & \multicolumn{2}{|c|}{ WHA } & \multicolumn{2}{|c|}{ WEA } \\
\hline & & & & & & Whole & $\% / \mathbf{m}$ & Whole & $\% / \mathrm{m}$ \\
\hline \multirow{3}{*}{$\begin{array}{l}\text { Wayne, } \\
1976\end{array}$} & \multirow{3}{*}{$\begin{array}{c}\text { Adams } \\
\text { Beach, } \\
\text { Florida, USA }\end{array}$} & \multirow{3}{*}{$\begin{array}{l}\text { Micro- } \\
\text { tidal }\end{array}$} & \multirow[t]{3}{*}{$\mathrm{NC}$} & \multirow{2}{*}{\multicolumn{2}{|c|}{ S. alterniflora }} & $71 \% / 20 \mathrm{~m}$ & 3.6 & $92 \% / 20 \mathrm{~m}$ & 4.6 \\
\hline & & & & & & & & & \\
\hline & & & & \multicolumn{2}{|c|}{ T. testudinum } & $42 \% / 20 \mathrm{~m}$ & 2.1 & $67 \% / 20 \mathrm{~m}$ & 3.4 \\
\hline $\begin{array}{l}\text { Knutson } \\
\text { et al., } \\
1982\end{array}$ & $\begin{array}{c}\text { Chesapeake } \\
\text { Bay, Virginia, } \\
\text { USA }\end{array}$ & $\begin{array}{l}\text { Micro- } \\
\text { tidal }\end{array}$ & $\begin{array}{l}\text { Wave } \\
\text { gage }\end{array}$ & \multicolumn{2}{|c|}{ S. alterniflora } & $\begin{array}{c}40 \% / 2.5 \mathrm{~m} \\
57 \% / 5 \mathrm{~m} \\
65 \% / 10 \mathrm{~m} \\
87 \% / 20 \mathrm{~m} \\
94 \% / 30 \mathrm{~m}\end{array}$ & 3.1 & $\begin{array}{c}64 \% / 2.5 \mathrm{~m} \\
72 \% / 5 \mathrm{~m} \\
88 \% / 10 \mathrm{~m} \\
98 \% / 20 \mathrm{~m} \\
100 \% / 30 \mathrm{~m}\end{array}$ & 3.33 \\
\hline $\begin{array}{l}\text { Moller et } \\
\text { al., } 1996\end{array}$ & $\begin{array}{c}\text { North } \\
\text { Norfolk, UK }\end{array}$ & $\begin{array}{l}\text { Macro- } \\
\text { tidal }\end{array}$ & PS. & \multicolumn{2}{|c|}{$\begin{array}{c}\text { North-West } \\
\text { European mixed } \\
\text { Saltmarsh }\end{array}$} & $\begin{array}{c}53.6 \% / 180 \\
\mathrm{~m}\end{array}$ & 0.3 & $79 \% / 180 \mathrm{~m}$ & 0.44 \\
\hline $\begin{array}{l}\text { Moller et } \\
\text { al., } 1999\end{array}$ & $\begin{array}{c}\text { North } \\
\text { Norfolk, UK }\end{array}$ & $\begin{array}{l}\text { Macro- } \\
\text { tidal }\end{array}$ & PS. & \multicolumn{2}{|c|}{$\begin{array}{c}\text { North-West } \\
\text { European mixed } \\
\text { Saltmarsh }\end{array}$} & $\begin{array}{c}60.96 \% / 18 \\
0 \mathrm{~m} \\
63 \% / 200 \mathrm{~m}\end{array}$ & 0.34 & $82 \% / 180 \mathrm{~m}$ & 0.45 \\
\hline \multirow[t]{2}{*}{$\begin{array}{c}\begin{array}{c}\text { Moller } \\
\text { and }\end{array} \\
\text { Spencer, } \\
2002\end{array}$} & \multirow[t]{2}{*}{$\begin{array}{c}\text { Dengie } \\
\text { Peninsula, } \\
\text { Essex, UK }\end{array}$} & \multirow[t]{2}{*}{$\begin{array}{c}\text { Macro- } \\
\text { tidal }\end{array}$} & \multirow[t]{2}{*}{ PS. } & \multirow{2}{*}{ 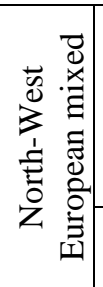 } & $\begin{array}{l}\text { Shallow } \\
\text { slopping } \\
\text { edge }\end{array}$ & $\begin{array}{c}87.37 \% / \\
163 \mathrm{~m}\end{array}$ & 0.54 & $\begin{array}{c}98.92 \% / \\
163 \mathrm{~m} \\
99 \% / 310 \mathrm{~m} \\
\text { (whole } \\
\text { transect) }\end{array}$ & $\begin{array}{l}0.61-0.3 \\
\text { (whole } \\
\text { transect) }\end{array}$ \\
\hline & & & & & $\begin{array}{c}\text { Cliffed } \\
\text { edge }\end{array}$ & $\begin{array}{c}43.81 \% / \\
10 \mathrm{~m}\end{array}$ & 4.38 & $\begin{array}{c}79.13 \% / \\
10 \mathrm{~m}\end{array}$ & 7.91 \\
\hline \multirow[t]{3}{*}{$\begin{array}{l}\text { Cooper, } \\
2005\end{array}$} & \multirow{3}{*}{$\begin{array}{l}\text { The Wash, } \\
\text { Eastern } \\
\text { England, UK }\end{array}$} & \multirow[t]{3}{*}{$\begin{array}{l}\text { Macro- } \\
\text { tidal }\end{array}$} & \multirow[t]{3}{*}{$\begin{array}{l}\text { Buoy and } \\
\text { PS. }\end{array}$} & \multicolumn{2}{|c|}{$\begin{array}{l}\text { Wrangle flats } \\
\text { transect }\end{array}$} & $\begin{array}{l}90.7 \% 1 \\
300 \mathrm{~m}\end{array}$ & 0.3 & $\begin{array}{l}97.1 \% / \\
300 \mathrm{~m}\end{array}$ & 0.32 \\
\hline & & & & \multicolumn{2}{|c|}{$\begin{array}{l}\text { Butterwick Low } \\
\text { transect }\end{array}$} & $\begin{array}{c}63.5 \% / \\
250\end{array}$ & 0.25 & $\begin{array}{l}71.9 \% / \\
250 \mathrm{~m}\end{array}$ & 0,29 \\
\hline & & & & \multicolumn{2}{|c|}{$\begin{array}{c}\text { Breast Sand } \\
\text { transect }\end{array}$} & $78 \% / 110 \mathrm{~m}$ & 0.7 & $\begin{array}{c}91.3 \% / \\
110 \mathrm{~m}\end{array}$ & 0.83 \\
\hline Moller, & Dengie & Macro- & PS. & \multicolumn{2}{|c|}{ North-West } & $2-7 \% / 10 \mathrm{~m}$ & $1-1.8$ & 1 & 1 \\
\hline
\end{tabular}




\begin{tabular}{|c|c|c|c|c|c|c|c|c|}
\hline 2006 & $\begin{array}{l}\text { Peninsula, } \\
\text { Essex, UK }\end{array}$ & tidal & & $\begin{array}{l}\text { European mixed } \\
\text { salt marsh }\end{array}$ & & & & \\
\hline $\begin{array}{l}\text { Yang et } \\
\text { al., } 2008\end{array}$ & $\begin{array}{c}\text { Eastern } \\
\text { Chongming, } \\
\text { China }\end{array}$ & $\begin{array}{l}\text { Macro- } \\
\text { tidal }\end{array}$ & $\begin{array}{l}\text { Wave } \\
\text { recorder / } \\
\text { PS. }\end{array}$ & $\begin{array}{l}\text { S. mariqueter, } S \text {. } \\
\text { alterniflora }\end{array}$ & $\begin{array}{l}16 \% / \\
16.5 \mathrm{~m}\end{array}$ & 0.95 & $\begin{array}{l}29 \% / \\
16.5 \mathrm{~m}\end{array}$ & 1,80 \\
\hline $\begin{array}{l}\text { Lambert, } \\
2009\end{array}$ & $\begin{array}{l}\text { Saint Laurent } \\
\text { Estuary, } \\
\text { Canada } \\
\end{array}$ & $\mathrm{NC}$ & PS. & $\begin{array}{l}\text { S. alterniflora, } S . \\
\text { patens }\end{array}$ & 1 & 1 & $\begin{array}{c}37-88 \% 1 \\
566 \mathrm{~m}\end{array}$ & $0.7-0.16$ \\
\hline \multirow{2}{*}{$\begin{array}{l}\text { Morgan } \\
\text { et al., } \\
2009\end{array}$} & \multirow[t]{2}{*}{$\begin{array}{l}\text { Northern New } \\
\text { England, USA }\end{array}$} & \multirow[t]{2}{*}{$\mathrm{NC}$} & \multirow[t]{2}{*}{ l } & S. alterniflora & $55 \% / 7 \mathrm{~m}$ & 7.86 & l & I \\
\hline & & & & $\begin{array}{c}\text { S. patens, } J . \\
\text { gerardii, } D . \\
\text { spicata and } P \text {. } \\
\text { maritima } \\
\end{array}$ & $52 \% / 7 \mathrm{~m}$ & 7.43 & 1 & 1 \\
\hline $\begin{array}{l}\text { Yang et } \\
\text { al., 2012 }\end{array}$ & $\begin{array}{c}\text { Eastern } \\
\text { Chongming, } \\
\text { China }\end{array}$ & $\begin{array}{l}\text { Macro- } \\
\text { tidal }\end{array}$ & PS. & S. alterniflora & $\begin{array}{l}30 \% / 7.5 \mathrm{~m} \\
51 \% / 20 \mathrm{~m} \\
79 \% / 51 \mathrm{~m}\end{array}$ & 2.34 & 1 & I \\
\hline $\begin{array}{l}\text { Moller et } \\
\text { al., } 2014\end{array}$ & ex situ & I & PS. & $\begin{array}{c}\text { Mixed saltmarsh } \\
\text { of } E \text {. athericus, } P \text {. } \\
\text { maritima, } A . \\
\text { prostrata } \\
\end{array}$ & $\begin{array}{l}20 \% / 40 \mathrm{~m} \\
\text { (high } \\
\text { energy } \\
\text { conditions) } \\
\end{array}$ & 0.5 & 1 & 1 \\
\hline \multirow{2}{*}{$\begin{array}{l}\text { Lechevali } \\
\text {-er, 2016* }\end{array}$} & \multirow{2}{*}{$\begin{array}{l}\text { Moëze, } \\
\text { France }\end{array}$} & \multirow{2}{*}{$\begin{array}{l}\text { Macro- } \\
\text { tidal }\end{array}$} & \multirow{2}{*}{$\begin{array}{l}\text { Wave } \\
\text { sensors / } \\
\text { PS. }\end{array}$} & S. maritima & I & 1.9 & I & I \\
\hline & & & & H. portulacoides & I & 4.6 & 1 & I \\
\hline \multirow[t]{3}{*}{$\begin{array}{l}\text { Collin et } \\
\text { al., 2018b }\end{array}$} & \multirow[t]{3}{*}{$\begin{array}{l}\text { Bay of } \\
\text { Lancieux, } \\
\text { France }\end{array}$} & \multirow[t]{3}{*}{$\begin{array}{l}\text { Mega- } \\
\text { tidal }\end{array}$} & \multirow[t]{3}{*}{ PS. } & $\begin{array}{l}\text { Lower marsh of } \\
\text { S. anglica and } S \text {. } \\
\text { europaea }\end{array}$ & $\begin{array}{c}5- \\
10 \% / 100 \mathrm{~m}\end{array}$ & $0.05-0.1$ & 1 & 1 \\
\hline & & & & $\begin{array}{l}\text { Medium marsh } \\
\text { of } H . \\
\text { portulacoides and } \\
\text { T. maritima } \\
\end{array}$ & $\begin{array}{c}10- \\
20 \% / 100 \mathrm{~m}\end{array}$ & $0.1-0.2$ & I & 1 \\
\hline & & & & $\begin{array}{c}\text { Upper marsh of } \\
\text { H. portulacoides, } \\
\text { T. maritima, } P . \\
\text { maritima and } F \text {. } \\
\text { rubra } \\
\end{array}$ & $\begin{array}{c}>20 \% / 100 \\
\mathrm{~m}\end{array}$ & $>0.2$ & l & l \\
\hline $\begin{array}{l}\text { Mury et } \\
\text { al., } 2018\end{array}$ & $\begin{array}{l}\text { BMSM, } \\
\text { France }\end{array}$ & $\begin{array}{c}\text { Mega- } \\
\text { tidal }\end{array}$ & PS. & $\begin{array}{c}\text { North-West } \\
\text { European mixed } \\
\text { Salt marsh }\end{array}$ & $\begin{array}{c}>78 \% / 100 \\
m\end{array}$ & $>0.78$ & 1 & I \\
\hline
\end{tabular}

\section{Mudflats/Sandflats}

The second part of the review work was dedicated to the WAS of mudflats and sandflats, in which seven studies were selected (Table 2). The majority of these studies indirectly dealt with the wave attenuation process by mudflats or sandflats. Indeed, mudflat transect measurements were mainly used as samples for comparison with the other "ecogeosystems" WAS (only Houser and Hill, 2010, focused on the mudflat WAS).

The WHA recorded in these various studies were generally found between $0.23 \% / \mathrm{m}$ (modest increase) and $0.2 \% / \mathrm{m}$ (with the exception of the Morgan et al., 2009, study data that reached $4 \% / \mathrm{m}$, a relatively high percentage due to the low distance between recording stations).

Table 2. Synthesis table of Mudflats and Sandflats wave attenuation service studies (WHA: Wave Height Attenuation; WEA: Wave Energy Attenuation; * grey literature, PS.: pressure sensors)

\begin{tabular}{|l|c|c|c|c|c|c|c|c|}
\hline \multirow{2}{*}{ Reference } & \multirow{2}{*}{$\begin{array}{c}\text { Study } \\
\text { Location }\end{array}$} & \multirow{2}{*}{$\begin{array}{c}\text { Tidal } \\
\text { Category }\end{array}$} & \multirow{2}{*}{ Materials } & \multirow{2}{*}{$\begin{array}{c}\text { Site } \\
\text { Characteristics }\end{array}$} & \multicolumn{4}{|c|}{ Wave Attenuation } \\
\cline { 5 - 8 } & & & Whole & \%/m & Whole & $\% / \mathbf{m}$ \\
\hline $\begin{array}{l}\text { Moller et } \\
\text { al., 1996. }\end{array}$ & $\begin{array}{c}\text { North } \\
\text { Norfolk, }\end{array}$ & $\begin{array}{c}\text { Macro- } \\
\text { tidal }\end{array}$ & PS. & $/$ & $\begin{array}{c}13.5 \% / \\
197 \mathrm{~m}\end{array}$ & 0.07 & $26 \% / 197 \mathrm{~m}$ & 0.13 \\
\hline $\begin{array}{l}\text { Moller et } \\
\text { al., 1999. }\end{array}$ & $\begin{array}{c}\text { North } \\
\text { Norfolk, } \\
\text { UK }\end{array}$ & $\begin{array}{c}\text { Macro- } \\
\text { tidal }\end{array}$ & PS. & $/$ & $15.29 \% /$ & 0.08 & $29 \% / 197 \mathrm{~m}$ & 0.15 \\
\hline
\end{tabular}




\begin{tabular}{|c|c|c|c|c|c|c|c|c|}
\hline \multirow{2}{*}{$\begin{array}{c}\begin{array}{c}\text { Moller } \\
\text { and }\end{array} \\
\text { Spencer, } \\
2002 \text {. }\end{array}$} & \multirow{2}{*}{$\begin{array}{c}\text { Dengie } \\
\text { Peninsula, } \\
\text { Essex, UK }\end{array}$} & \multirow{2}{*}{$\begin{array}{c}\text { Macro- } \\
\text { tidal }\end{array}$} & \multirow[t]{2}{*}{ PS. } & $\begin{array}{c}\text { Marsh edge : } \\
\text { shallow } \\
\text { slopping } \\
\end{array}$ & $\begin{array}{c}20.57 \% / \\
147 \mathrm{~m} \\
\end{array}$ & 0.14 & $\begin{array}{l}35.25 \% / \\
147 \mathrm{~m}\end{array}$ & 0.24 \\
\hline & & & & $\begin{array}{l}\text { Marsh edge : } \\
\text { cliffed edge }\end{array}$ & $\begin{array}{c}+23.91 \% / \\
102 \mathrm{~m} \\
\end{array}$ & +0.23 & $\begin{array}{c}+55.06 \% / \\
102 \mathrm{~m} \\
\end{array}$ & +0.54 \\
\hline \multirow{3}{*}{$\begin{array}{c}\text { Cooper, } \\
2005 .\end{array}$} & \multirow{3}{*}{$\begin{array}{l}\text { The Wash, } \\
\text { Eastern } \\
\text { England, } \\
\text { UK }\end{array}$} & \multirow{3}{*}{$\begin{array}{l}\text { Macro- } \\
\text { tidal }\end{array}$} & \multirow{3}{*}{$\begin{array}{c}\text { Buoy / } \\
\text { PS. }\end{array}$} & $\begin{array}{c}\text { Wrangle flats } \\
\text { transect }\end{array}$ & $10.1 \% / 330 \mathrm{~m}$ & 0.03 & $15.9 \% / 330 \mathrm{~m}$ & 0.05 \\
\hline & & & & $\begin{array}{c}\text { Butterwick } \\
\text { Low transect }\end{array}$ & $23 \% / 390 \mathrm{~m}$ & 0.06 & $35.8 \% / 390 \mathrm{~m}$ & 0.09 \\
\hline & & & & $\begin{array}{l}\text { Breast Sand } \\
\text { transect }\end{array}$ & $36.4 \% / 180 \mathrm{~m}$ & 0.2 & $56.2 \% / 180 \mathrm{~m}$ & 0.31 \\
\hline $\begin{array}{c}\text { Yang et } \\
\text { al., } 2008 .\end{array}$ & $\begin{array}{l}\text { Eastern } \\
\text { Chong- } \\
\text { ming, } \\
\text { China } \\
\end{array}$ & $\begin{array}{l}\text { Macro- } \\
\text { tidal }\end{array}$ & $\begin{array}{l}\text { Wave-tide } \\
\text { recorder / } \\
\text { PS. }\end{array}$ & I & $11 \% / 185 \mathrm{~m}$ & 0.06 & $21 \% / 185 \mathrm{~m}$ & 0.11 \\
\hline $\begin{array}{c}\text { Morgan } \\
\text { et al., } \\
2009 \text {. }\end{array}$ & $\begin{array}{l}\text { Northern } \\
\text { New } \\
\text { England, } \\
\text { USA } \\
\end{array}$ & $\mathrm{NC}$ & $\mathrm{NC}$ & I & $28 \% / 7 \mathrm{~m}$ & 4.0 & / & I \\
\hline $\begin{array}{c}\text { Houser } \\
\text { and Hill, } \\
2010 .\end{array}$ & $\begin{array}{l}\text { Fraser } \\
\text { River } \\
\text { Delta, } \\
\text { Canada }\end{array}$ & $\mathrm{NC}$ & $\begin{array}{l}\text { Current } \\
\text { profiler / } \\
\text { PS. }\end{array}$ & I & $42 \% / 4500 \mathrm{~m}$ & 0.01 & $62 \% / 4500 \mathrm{~m}$ & 0.014 \\
\hline
\end{tabular}

\section{Seagrasses}

Concerning the review work allocated to seagrasses WAS, an important methodological choice has been made by excluding all studies dealing with artificial seagrasses (Table 3). Indeed, considering the relative fragility of seagrasses in their natural environment, a plethora of studies focused on artificial seagrasses. The exclusion of artificial seagrasses from this review is justified by primarily targeting the natural components of the intertidal domain and their responses to meteorological and wave forcing. Considering the studies dealing with WHA, a great variability has been recorded, from $0.03 \% / \mathrm{m}$ to $0.77 \% / \mathrm{m}$. This variability in results can be explained by the fragility of this kind of ecosystem and his dependence on the experimental conditions. Furthermore, another explanatory factor of this variability is the diversity of the morphological characteristics (e.g., root system, leaf morphometry) of the various species.

Table 3. Synthesis table of Seagrasses wave attenuation service studies (WHA: Wave Height Attenuation; WEA: Wave Energy Attenuation; * grey literature, PS.: pressure sensors)

\begin{tabular}{|c|c|c|c|c|c|c|c|c|}
\hline \multirow{3}{*}{ Reference } & \multirow{3}{*}{$\begin{array}{l}\text { Study } \\
\text { Location }\end{array}$} & \multirow{3}{*}{$\begin{array}{c}\text { Tidal } \\
\text { category }\end{array}$} & \multirow{3}{*}{ Materials } & \multirow{3}{*}{$\begin{array}{c}\text { Site } \\
\text { characteristics }\end{array}$} & \multicolumn{4}{|c|}{ Wave attenuation } \\
\hline & & & & & \multicolumn{2}{|c|}{ WHA } & \multicolumn{2}{|c|}{ WEA } \\
\hline & & & & & whole & $\% / \mathrm{m}$ & whole & $\% / \mathrm{m}$ \\
\hline $\begin{array}{c}\text { Fonseca } \\
\text { and } \\
\text { Cahalan, } \\
1992\end{array}$ & $\begin{array}{l}\text { ex situ - } \\
\text { wave } \\
\text { flume }\end{array}$ & l & $\begin{array}{l}\text { Wave } \\
\text { flume }\end{array}$ & $\begin{array}{c}\text { Halodule } \\
\text { wrightii, } \\
\text { Syringodium } \\
\text { filiforme, } \\
\text { Thalassia } \\
\text { testudinum, } \\
\text { Zostera marina }\end{array}$ & I & I & $20-76 \% / 1 \mathrm{~m}$ & an $40 \%)$ \\
\hline $\begin{array}{c}\text { Prager } \\
\text { and } \\
\text { Halley, } \\
1999\end{array}$ & $\begin{array}{c}\text { Calusa } \\
\text { Key, } \\
\text { Florida } \\
\text { Bay, USA }\end{array}$ & $\mathrm{NC}$ & PS. & $\begin{array}{l}\text { Thalassia } \\
\text { testudinum }\end{array}$ & l & l & $\begin{array}{c}>80 \% / 2500 \\
\mathrm{~m}\end{array}$ & 0.03 \\
\hline
\end{tabular}




\begin{tabular}{|c|c|c|c|c|c|c|c|c|c|}
\hline \multirow{2}{*}{$\begin{array}{c}\text { Paul and } \\
\text { Amos, } \\
2011\end{array}$} & \multirow{2}{*}{$\begin{array}{c}\text { Ryde } \\
\text { Sand, Isle } \\
\text { of Wight, } \\
\text { UK }\end{array}$} & \multirow{2}{*}{$\begin{array}{l}\text { Macro- } \\
\text { tidal }\end{array}$} & \multirow{2}{*}{ PS. } & \multirow{2}{*}{$\begin{array}{c}\text { Zoster } \\
-a \\
\text { noltii }\end{array}$} & $\begin{array}{l}\text { High } \\
\text { shoot } \\
\text { density }\end{array}$ & $\begin{array}{c}20 \% / 30- \\
95 \mathrm{~m}\end{array}$ & $0.66-0.21$ & I & I \\
\hline & & & & & $\begin{array}{l}\text { Low } \\
\text { shoot } \\
\text { density }\end{array}$ & $\begin{array}{c}<10 \% / 30- \\
95 \mathrm{~m}\end{array}$ & $0.33-0.11$ & 1 & I \\
\hline $\begin{array}{c}\text { Bradley } \\
\text { and } \\
\text { Houser, } \\
2009\end{array}$ & $\begin{array}{c}\text { Santa } \\
\text { Rosa } \\
\text { Island, } \\
\text { Florida, } \\
\text { USA }\end{array}$ & $\begin{array}{l}\text { Micro- } \\
\text { tidal }\end{array}$ & PS. & $\begin{array}{l}\text { That } \\
\text { testu }\end{array}$ & $\begin{array}{l}\text { assia } \\
\text { dinum }\end{array}$ & $\begin{array}{c}+20 \% / 5^{\text {th }} \\
\text { m then } \\
\text { decrease of } \\
30 \% / 39 \mathrm{~m}\end{array}$ & $\begin{array}{c}0.77 \\
(39 \mathrm{~m})\end{array}$ & I & I \\
\hline $\begin{array}{l}\text { Christian- } \\
\text { en et al., } \\
2013\end{array}$ & $\begin{array}{l}\text { Derawan } \\
\text { Island, } \\
\text { Indonesia }\end{array}$ & $\mathrm{NC}$ & PS. & $\begin{array}{l}\text { Hal } \\
\text { unin }\end{array}$ & $\begin{array}{l}\text { dule } \\
\text { ervis }\end{array}$ & $\begin{array}{l}18 \% / 667 \mathrm{~m} \\
30 \% / 450 \mathrm{~m} \\
11 \% / 200 \mathrm{~m}\end{array}$ & 0.03-0.07 & I & I \\
\hline
\end{tabular}

\section{Oyster reefs}

Oyster reefs are deemed as natural breakwaters since several centuries and are currently utilized in ecosystem restoration programs. Their value as a protective ecosystem is attested, but there are surprisingly not so many studies, which aimed at quantifying their WAS value. Among the retained studies, three out of four are ex situ studies (Table 4). Data from these studies were difficult to harmonize because of the discrete shape of the oyster reefs, thus an attenuation percentage per meter (i.e., surface metrics) could be inappropriate to estimate it. A punctual metric would be more likely to capture the WAS of these reefs. The only value we could find stemmed from Garvis (2012): $23 \%$ attenuation for a three-metre transect, that is to say $7.6 \% / \mathrm{m}$. Noteworthy was the absence of consideration of the non-linearity of the attenuation and the possible shoaling effect.

Table 4. Synthesis table of Oyster Reefs wave attenuation service studies (WHA: Wave Height Attenuation; WEA: Wave Energy Attenuation; * grey literature, PS.: pressure sensors)

\begin{tabular}{|c|c|c|c|c|c|c|c|c|}
\hline \multirow{3}{*}{ Reference } & \multirow{3}{*}{$\begin{array}{c}\text { Study } \\
\text { Location }\end{array}$} & \multirow{3}{*}{$\begin{array}{c}\text { Tidal } \\
\text { category }\end{array}$} & \multirow{3}{*}{ Materials } & \multirow{3}{*}{$\begin{array}{c}\text { Site } \\
\text { characteristics }\end{array}$} & \multicolumn{4}{|c|}{ Wave attenuation } \\
\hline & & & & & \multicolumn{2}{|c|}{ WHA } & \multicolumn{2}{|c|}{ WEA } \\
\hline & & & & & whole & $\% / \mathrm{m}$ & whole & $\% / \mathrm{m}$ \\
\hline \multirow{3}{*}{$\begin{array}{l}\text { Garvis, } \\
\text { 2012* }^{*}\end{array}$} & \multirow{3}{*}{ ex situ } & \multirow{3}{*}{ I } & \multirow{3}{*}{ PS. } & Dead reef & $<1 \% / 3 \mathrm{~m}$ & & 1 & 1 \\
\hline & & & & Natural reef & $23 \% / 3 \mathrm{~m}$ & & 1 & 1 \\
\hline & & & & Restored reef & $25 \% / 3 \mathrm{~m}$ & & I & l \\
\hline $\begin{array}{l}\text { Taube, } \\
2013 *\end{array}$ & $\begin{array}{c}\text { Delmarva } \\
\text { Peninsula, } \\
\text { Virginia, } \\
\text { USA }\end{array}$ & $\begin{array}{l}\text { Micro- } \\
\text { tidal }\end{array}$ & $\begin{array}{c}\text { Wave } \\
\text { recorder - } \\
\text { Acoustic } \\
\text { Doppler }\end{array}$ & $\begin{array}{l}\text { Crassostrea } \\
\text { virginica } \text { reef }\end{array}$ & I & I & $49 \%$ & l \\
\hline \multirow{2}{*}{$\begin{array}{l}\text { Manis et } \\
\text { al., } 2015\end{array}$} & \multirow[b]{2}{*}{ ex situ } & \multirow[b]{2}{*}{ I } & \multirow[b]{2}{*}{ PS. } & $\begin{array}{l}\text { Newly deployed } \\
\text { oyster shell }\end{array}$ & $10.3 \%$ & I & $18.7 \%$ & I \\
\hline & & & & $\begin{array}{c}\text { 1-year } \\
\text { established } \\
\text { oyster }\end{array}$ & $10.5 \%$ & I & $44.7 \%$ & I \\
\hline $\begin{array}{l}\text { Godfroy, } \\
2017 *\end{array}$ & ex situ & I & Model & I & $9-64 \%$ & I & I & I \\
\hline
\end{tabular}

\section{Shelly cheniers}

The capacity of shelly cheniers to alleviate wave energy is also accepted, although it is little documented. Shelly cheniers are besides relatively rare geomorphic structures worldwide, resulting in a few studies about their WAS. To date, we listed only two articles about this attenuation aspect (Table 5). The first study showed a very high WEA rate, around $98 \%$ for healthy cheniers, whilst the second study, focused on the WHA, showed a rate around $61 \%$ for the whole well-developed chenier, and around $39 \%$ for the degraded one. It is also advocated to use a global punctual rate for the whole reef, since the attenuation percentage per meter looks inappropriate (discrete aspect). 
Table 5. Synthesis table of Shelly Cheniers wave attenuation service studies (WHA: Wave Height Attenuation; WEA: Wave Energy Attenuation; * grey literature, PS.: pressure sensors)

\begin{tabular}{|c|c|c|c|c|c|c|c|c|}
\hline \multirow{3}{*}{ Reference } & \multirow{3}{*}{$\begin{array}{c}\text { Study } \\
\text { Location }\end{array}$} & \multirow{3}{*}{$\begin{array}{c}\text { Tidal } \\
\text { category }\end{array}$} & \multirow{3}{*}{ Materials } & \multirow{3}{*}{$\begin{array}{c}\text { Site } \\
\text { characteristics }\end{array}$} & \multicolumn{4}{|c|}{ Wave attenuation } \\
\hline & & & & & \multicolumn{2}{|c|}{ WHA } & \multicolumn{2}{|c|}{ WEA } \\
\hline & & & & & whole & $\% / m$ & whole & $\% / \mathrm{m}$ \\
\hline \multirow{2}{*}{$\begin{array}{l}\text { Jeanson } \\
\text { et al., } \\
2016\end{array}$} & \multirow{2}{*}{$\begin{array}{l}\text { BMSM, } \\
\text { France }\end{array}$} & \multirow{2}{*}{$\begin{array}{l}\text { Mega- } \\
\text { tidal }\end{array}$} & \multirow{2}{*}{ PS. } & $\begin{array}{l}\text { Well-developed } \\
\text { shelly cheniers }\end{array}$ & I & I & $98 \%$ & I \\
\hline & & & & $\begin{array}{l}\text { Degraded shelly } \\
\text { cheniers }\end{array}$ & I & I & $70 \%$ & I \\
\hline \multirow{2}{*}{$\begin{array}{l}\text { Mury et } \\
\text { al., } 2018\end{array}$} & \multirow{2}{*}{$\begin{array}{l}\text { BMSM, } \\
\text { France }\end{array}$} & \multirow{2}{*}{$\begin{array}{l}\text { Mega- } \\
\text { tidal }\end{array}$} & \multirow{2}{*}{ PS. } & $\begin{array}{l}\text { Well-developed } \\
\text { shelly cheniers }\end{array}$ & $\begin{array}{c}61.1 \% / 100 \\
\mathrm{~m}\end{array}$ & 0.61 & l & I \\
\hline & & & & $\begin{array}{l}\text { Degraded shelly } \\
\text { cheniers }\end{array}$ & $\begin{array}{c}39.3 \% / 100 \\
\mathrm{~m}\end{array}$ & 0.39 & I & I \\
\hline
\end{tabular}

\section{Honeycomb worm and sandworm reefs}

To our knowledge, WAS of honeycomb worm (Sabellaria alveolota) and sandworm (Lanice conchilega) reefs were not studied yet. Nevertheless, literature references evoke a potential mitigation process by the honeycomb colonies, which might significantly increase the surface roughness (Collin et al., 2018a) and so reduce wave energy. This lack of consideration of engineer-ecosystems into their protective role could be explained for several reasons. These biogenic structures are overall poorly known due to their relative rarity in comparison with some others features like saltmarshes or seagrasses. In addition, their location at the bottom of the intertidal zone (remote from the coastline) drives scientists to consider them as some marginal elements in the WAS of the entire intertidal zone, especially in a mega-tidal environment.

\section{Discussion}

This review of the existing literature about the WAS of the intertidal "ecogeosystems" in temperate areas allowed us to highlight several elements.

Indeed, it appears that WAS of some "ecogeosystems" are well documented, as it is the case for saltmarshes (Table 1). Contrariwise, some features, rarer worldwide or located lower in the intertidal zone, are poorly known, particularly shelly cheniers, honeycomb worm and sandworm reefs.

Thus, by a quantitative and qualitative analysis of the different papers, we can deduce one pattern: the more we go away from the coastline, the more we observe gaps in the WAS knowledge. The distribution of the reviewed papers along a cross-shore transect could be used to illustrate this finding (Figure 6).

Another element we drew from this review is the difficulties to harmonize the various data across studies. These difficulties are due to several factors. The first one is about the structural heterogeneity of study sites and

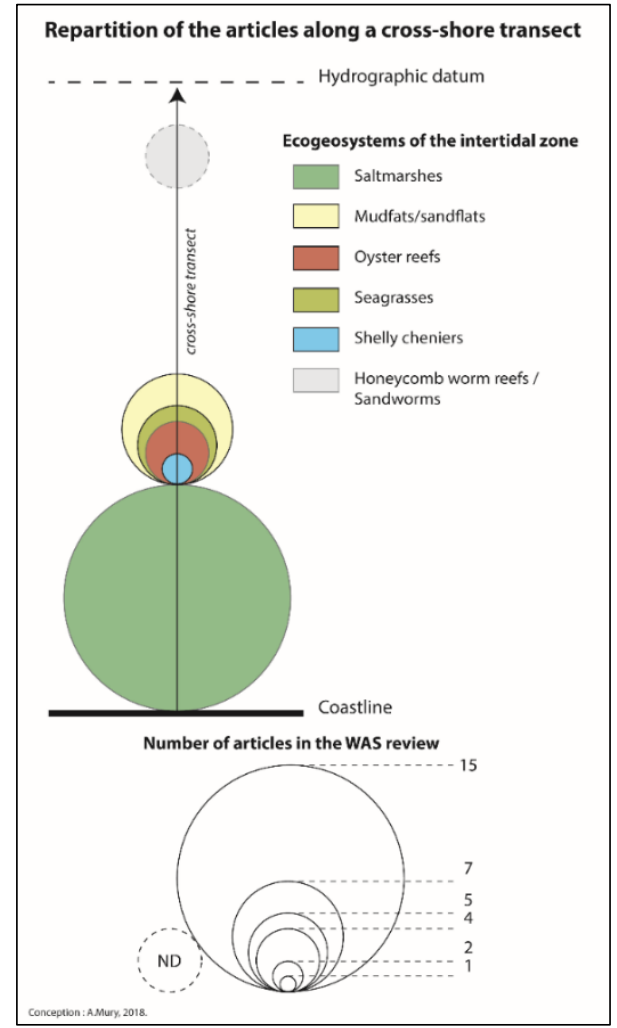

Figure 6. Scheme of papers' distribution along a conceptualized cross-shore transect

"ecogeosystems": their shape (punctual features as cheniers versus surface features as saltmarshes), and their inherent composition (i.e., different vegetation communities for saltmarshes studies, various length measurement transects and various measurement conditions: weather, seasons, tidal category, water levels). The second reason is about the published datasets: some technical information might miss; foreshore and seabed morphometry and features backing the natural barriers, hydrodynamic regime and distance from the coastline are often not specified, thus introducing 
biases in the data harmonization due to the ignorance of the influence of these elements on the WAS. The third reason concerns the heterogeneity of measurement methods between the different studies (pressure sensor, buoy, wave recorder), which complexify and even impede the comparison between studies. Moreover, significant wave height calculation methods are overshadowed, because of the absence of specifications in papers.

As a single variable based on significant wave height WHA might appear as a simplistic methodological choice in this review. Not considered in this review were the characteristics linked to the tidal regime (infragravity waves) and the flow velocity, taking probably part of the marine flood hazards. Moreover, in most cases, WHA is mathematically approached as a linear process along the study section while it is obviously a non-linear process in the complex real world (Koch et al., 2009). The wave reduction decreases from the start to the end of the section, i.e., the shoaling effect, which is rarely investigated in the research studies. This simplified linear approach of the mitigation process could be explained by the methodological and material contingencies. Indeed, frequently the data used for the calculation of the wave attenuation arise from punctual and fixed stations, at the beginning and the end of the study section. This approach does not allow to observe the process in its entirety. Several technical processes, in on-going development, should correct for this problem, in particular by the contribution of the high-resolution satellite imagery (Collin et al., 2018b).

Table 6 synthetizes results obtained from the papers reviewed. Despite its plain design, the table gives a first overview of the WAS value for every studied "ecogeosystem".

Table 6. Synthesis table of WAS from the meta-analysis (WHA: Wave Height Attenuation, * Wave Energy Attenuation data were used for Shelly Cheniers)

\begin{tabular}{|c|c|c|}
\hline Shape & Ecogeosystems & WHA \\
\hline \multirow{2}{*}{ Surface } & Saltmarshes & $0.05-7.86 \% / \mathrm{m}$ \\
\cline { 2 - 3 } & Mudflats & $0.01-4 \% / \mathrm{m}$ \\
\cline { 2 - 3 } & Seagrasses & $0.03-0.77 \% / \mathrm{m}$ \\
\hline Punctual & Oyster reefs & $\begin{array}{c}1-64 \%(\text { whole } \\
\text { reef) }\end{array}$ \\
\cline { 2 - 3 } & Shelly Cheniers & $\begin{array}{c}70-98 \%(\text { whole } \\
\text { transect)* }\end{array}$ \\
\hline
\end{tabular}

To conclude, a work of harmonization of the results of the different studies is recommended by insisting on the transparency in the methods used. To leverage a generic model adaptable for all coastlines with their diversity of "ecogeosystems" and specificities, we propose to improve the knowledge about all the potential "ecogeosystems" WAS worldwide. To solve the problem of harmonization, a robust approach would be to either find generic metrics across all "ecogeosystems" or ranking them through a table showing both range, mean and standard deviation values. This synoptic product holds great promise to be integrated into toolboxes of stakeholders tasked with nature-based mitigation of coastal risks.

\section{References}

Bradley, K. and Houser, C. 2009. "Relative velocity of seagrass blades: implication for wave attenuation in low-energy environments", Journal of Geophysiscal Research, 114(F1).

Christianen, M.J.A., van Belzen, J., Herman, J., van Katwijk, M.M., Lamers, L.P.M., van Leent, P.J.M. and Bouma, T.J. 2013. "Low-canopy seagrass beds still provide important coastal protection services", PLoS ONE, 8(5), e62413.

Collin, A., Dubois, S., Ramambason, C. and Etienne, S. 2018a. "Very high-resolution mapping of emerging biogenic reefs using airborne optical imagery and neural network: the honeycomb worm (Sabellaria alveolata) case study", International Journal of Remote Sensing, 1-16.

Collin, A., Lambert, N., James, D. and Etienne, S. 2018b. "Mapping wave attenuation induced by salt marsh vegetation using WorldView-3 satellite imagery", Revista de investigación Marina, 25(2), 67-69.

Cooper, N.J. 2005. "Wave dissipation across Intertidal surfaces in the wash tidal inlet, Eastern England", Journal of Coastal Research, 21(1), 28-40.

Fonseca, M.S. and Cahalan, J.A. 1992. "A preliminary evaluation of wave attenuation by four species of seagrass", Estuarine, coastal and shelf science, 35, 564-576.

Garvis, S.K. 2012. "Quantifying the impacts of oyster reef restoration on oyster coverage, wave attenuation and seagrass fragment retention in Mosquito Lagoon, Florida", Msc. Thesis, University of Central Florida, Electronic These and dissertations. 2284.

Godfroy, M. 2017. "Quantifying wave attenuation by nature based solutions in Galveston bay", Msc.Thesis, Delft University of Technology, pp. 145.

Harris, D.L., Rovere, A., Parravicini, V., Casella, E., Canavesio, R. and Collin, A. 2016. "Healthy coral reefs may assure coastal protection in face of climate change related sea level rise", American Geophysical Union Fall Meeting, GC11D-1167.

Harris, D.L., Rovere, A., Casella, E., Power, H., Canavesio, R., Collin, Pomeroy, A., Webster, JM and Parravicini, V. 2018. "Coral reef structural complexity provides important coastal protection from waves under rising sea levels", Science advances, 4(2), eaao4350. 
Houser, C. and Hill, P. 2010. "Wave attenuation across an Intertidal sand flat: Implication for mudflat development", Journal of Coastal Research, 26(3), 403-411.

Jeanson, M., Etienne, S. and Collin, A. 2016. "Wave attenuation and Coastal Protection by Shelly Ridges: Mont-Saint-Michel Bay, France", Journal of Coastal Research, 75, 398-402.

Knutson, P.L., Brochu, R.A., Seelig, W.N. and Inskeep, M. 1982. "Wave damping in spartina alterniflora", Wetlands, 2, 87-104.

Koch, E.W., Barbier, E.B., Siliman, B.R., Reed, D.J., Perillo, G.M.E., Granek E.F., Primavera, J.H., Muthiga, N., Polasky, S., Halpern, B.S., Kennedy, C.J., Kappel, C.V. and Wolanski, E. 2009. "Non-linearity in ecosystem services: temporal and spatial variability in coastal protection", Frontiers in Ecology and the Environment, 7, 29-37.

Lambert, N. 2009. «Modélisation de la dissipation de l'énergie des vagues par la végétation de marais littoral ». PhD thesis. Rimouski, Québec, Université du Québec à Rimouski, Institut des sciences de la mer de Rimouski, pp. 97.

Lechevalier, A. 2016. «Etude de l'atténuation des vagues par un champ de végétation composé de Spartina maritima et Halimione portulacoides », Msc. Thesis, Université de La Rochelle, pp. 34.

Manis, J.E., Garvic, S.K., Jachec, S.M. and Walters, L.J. 2015. "Wave attenuation experiments over living shorelines over time: a wave tank study to assess recreational boating pressure", Journal of Coastal Conservation, planning and management, 19(1), 1-11.

Moller, I., Spencer, T. and French, J.R. 1996. "Wind wave dissipation over saltmarshes surfaces: preliminary results from Norfolk, England", Journal of Coastal Research, 12(4), 1009-1016.

Moller, I., Spencer, T., French J.R., Leggett D.J. and Dixon M. 1999. "Wave transformation over salt marshes: a field and numerical modelling study from North Norfolk, England", Estuarine, Coastal and Shelf Science, 49, 411-426.

Moller, I. and Spencer, T. 2002. "Wave dissipation over macro-tidal saltmarshes: effects of marshes edge typology and vegetation change", Journal of coastal research, 506-521.

Moller, I. 2006. "Quantifying saltmarsh vegetation and its effect on wave height dissipation: Result from a UK East coast saltmarsh", Estuarine Coastal Shelf Science, 69, 337-351.

Moller, I., Kudella, M., Rupprecht, F., Spencer, T., Paul, M., van Wesenbeeck B.K., Wolters, G., Jensen, K., Bouma, T.J., Miranda-Lange, M. and Schimmels, S. 2014. "Wave attenuation over coastal salt marshes under storm surge conditions", Nature Geoscience, 7, 727-731.

Morgan, P.A., Burdick, D.M. and Short F.T. 2009. "The functions and values of fringing salt marshes in Northern New England, USA", Estuaries and Coasts, 32, 483-495.

Mury, A., Collin, A. and Etienne, S. 2018. "Wave attenuation service of saltmarshes and shelly cheniers: a spatio-temporal study in Mont-Saint-Michel Bay, France”, Geophysical Research Abstracts, 20, 2044.

Paul, M. and Amos, C.L. 2011. "Spatial and seasonal variation in wave attenuation over Zostera noltii", Journal of geophysical research, 116, C08019.

Prager, E.J. and Halley, R.B. 1999. "The influence of seagrass on shell layers and Florida Bay mudbanks", Journal of Coastal Research, 15(4), 1151-1162.

Taube, S.R. 2013. "Impact of fringing oyster reefs on wave attenuation and marsh erosion rates." Msc. Thesis, University of Virginia.

Wayne, C.J. 1976. "The effects of sea and marsh grass on wave energy", Coastal Research Notes, 14, 6-8.

Yang, S.L., Li, H., Ysebaert, T., Bouma, T.J., Zhang, W.X., Wang, Y.Y., Li, P., Li M. and Ding, P.X. 2008. "Spatial and temporal variations in sediment grain size in tidal wetlands, Yangtze Delta: On the role of physical and biotic controls", Estuarine, Coastal and Shelf Sciences, 77, 657-671.

Yang, S.L., Shi B.W., Bouma, T.J. and Ysebaert, T. 2012. "Wave attenuation at a salt marsh margin: a case study of an exposed coast on the Yangtze Estuary", Estuaries and Coasts, 35, 169-182. 